\title{
Interfacial Haloplumbate Modulation for Efficient and Stable Hybrid Perovskite Photovoltaics
}

Huanhuan Wang ${ }^{1 \dagger}$, Zhuang Zhang ${ }^{1 \dagger}$, Jovana V. Milić ${ }^{2,5}$, Liguo Tan $^{3}$, Zaiwei Wang ${ }^{4}$, Rong Chen $^{1}$, Xin Jing $^{1}$, Chenyi $\mathrm{Yi}^{3}$, Yi Ding ${ }^{1}$, Yuelong $\mathrm{Li}^{1}$, Ying Zhao ${ }^{1}$, Xiaodan Zhang ${ }^{1}$, Anders Hagfeldt ${ }^{4}$, Michael Grätzel $^{2}$, Jingshan Luo ${ }^{1 *}$

${ }^{1}$ Institute of Photoelectronic Thin Film Devices and Technology, Solar Energy Research Center, Key Laboratory of Photoelectronic Thin Film Devices and Technology of Tianjin, Ministry of Education Engineering Research Center of Thin Film Photoelectronic Technology, Renewable Energy Conversion and Storage Center, Nankai University, Tianjin 300350, China

${ }^{2}$ Laboratory of Photonics and Interfaces, Institute of Chemical Sciences and Engineering, Ecole Polytechnique Fedérale de Lausanne (EPFL), 1015 Lausanne, Switzerland

${ }^{3}$ State Key Laboratory of Power System, Department of Electrical Engineering, Tsinghua University, Beijing, China

${ }^{4}$ Laboratory of Photomolecular Science, Institute of Chemical Sciences Engineering, Ecole Polytechnique Fedérale de Lausanne (EPFL),1015 Lausanne, Switzerland

${ }^{5}$ Adolphe Merkle Institute, University of Fribourg, 1700 Fribourg, Switzerland

${ }^{\dagger}$ These authors contribute equally to this work.

*Email: jingshan.luo@nankai.edu.cn

\begin{abstract}
The commercialization of perovskite solar cells is mainly limited by their operational stability. Interlayer modification by thin interface materials between the perovskite and the charge transport layers is one of the most effective methods to promote the efficiency and stability of perovskite devices. However, the commonly used interlayer materials do not meet all the demands, including good film quality, excellent stability, and passivation capability without interfering with the charge transport. In this work, we propose a new material, water stable haloplumbate [TBA]Pbl ${ }_{3}$ for interfacial modification, which is formed on the perovskite surface in-situ by tetra-butylammonium iodide (TBAI) treatment. Benefiting from its passivation effect and robustness, the modified devices result in a power conversion efficiency of $22.90 \%$ with excellent environmental and operational stability.
\end{abstract}

Key words: Interlayer modification, haloplumbates, water-stable, perovskite solar cells

Introduction

Perovskite solar cells have shown great promise for photovoltaic applications due to their low cost 
and high power conversion efficiency (PCE).$^{1-7}$ Their PCE has increased from $3.8 \%{ }^{8}$ to certified $25.5 \%{ }^{9}$ in the past decade, ascribed to their excellent optical and electronic properties, such as tunable bandgap, high absorption coefficient, and long charge carrier lifetime. ${ }^{2,10-12}$ However, the instability of perovskite materials still restricts their large-scale commercialization. ${ }^{1,13-15}$ This is the result of multiple factors, such as the reactivity to moisture, oxygen, thermal stress, voltage bias, and light, which cause the unexpected degradation of PSCs. ${ }^{16-18}$

In recent years, interfacial engineering of thin interlayers on the surface of the perovskite layer is proved to be one of the most effective methods for improving the stability of PSCs. ${ }^{19}$ This has involved various molecular assemblies ${ }^{20-23}$ as well as graphene composites ${ }^{24}$, along with lowdimensional perovskites ${ }^{25,26}$, polymers ${ }^{27}$, and inorganic materials ${ }^{28}$. An ideal interfacial modulator should fulfill several requirements, namely: (i) forming a well-defined compact structure with good coverage, (ii) featuring excellent stability against environmental and operational conditions, with (iii) appropriate energy alignment, along with the (iv) passivation capacity. A compact and well-covered interlayer can prevent water and oxygen from damaging the perovskite layer underneath, while preventing the volatile species from being released, whereas the interlayer itself should be stable against moisture, oxygen, thermal, and other operating factors to provide long-term protection. Finally, the interlayer should ideally have a good energy level alignment, so that it would not limit the charge carrier extraction, while it should also be able to passivate defects on the surface of the perovskite layer, which could contribute to the overall photovoltaic performance.

Haloplumbates are low-dimensional organolead halide complexes which contain a lead halide $\left[\mathrm{Pb}_{n} \mathrm{X}_{\mathrm{m}}\right]$ octahedral framework different from the one in hybrid halide perovskites. They have been studied due to their diverse crystal structure ${ }^{29-33}$, luminescence ${ }^{33-36}$ and thermal stability ${ }^{29,31,37}$. Recently, $\left[\mathrm{Pb}_{8} \mathrm{I}_{8}\left(\mathrm{H}_{2} \mathrm{O}\right)_{3}\right]^{8+}\left[\mathrm{O}_{2} \mathrm{C}\left(\mathrm{CH}_{2}\right)_{4} \mathrm{CO}_{2}^{-}\right]_{4}$ haloplumbate was used for photocatalytic water splitting, showing excellent photoelectrical properties and superior moisture resistance. ${ }^{39}$ Tetrabutylammonium lead iodide $\left([\mathrm{TBA}] \mathrm{Pbl}_{3}\right)$ is another type haloplumbate with excellent stability in water and under thermal stress. ${ }^{28,38}$ Based on these unique properties, haloplumbates are potential candidates for interfacial modulators of hybrid perovskite devices.

$\mathrm{TBA}^{+}$has been reported to assist crystallization and film formation to improve photovoltaic performance and act as hydrophobic materials to promote stability with formation of lowdimensional materials when used as additives, ${ }^{40-44}$ and most recently, as post-treatment materials ${ }^{45,46}$ during the submission of this paper. Inspired by these results, it is possible to form [TBA]Pbl ${ }_{3}$ on the surface of perovskite with TBAl treatment. In this work, we systematically studied the properties of [TBA] $\mathrm{Pbl}_{3}$ and introduced it on the surface of the perovskite layer through an in-situ reaction between the perovskite and TBAI, which was found to be self-limiting and well-defined on the surface of perovskite layer without affecting the underlayer while significantly reducing the trap density. As a result, we obtained solar cells with efficiencies up to $22.90 \%$ and outstanding stability against moisture and heat under operating conditions.

\section{Results and discussion}


The haloplumbate single crystal of [TBA] $\mathrm{Pbl}_{3}$ was isolated through slow evaporation process of the precursor solution of $\mathrm{Pbl}_{2}$ and TBAI (Figure 1a; experimental details provided in the supporting information), forming yellow rod-like crystals (Figure S1). The X-ray diffraction (XRD) pattern of the crystal is identical with the calculated $[\mathrm{TBA}] \mathrm{Pbl}_{3}$ pattern (Figure 1b), evidencing its formation. It crystallizes in tetragonal space group I-42d with a 1D face-sharing inorganic chain (Figure 1a).

a

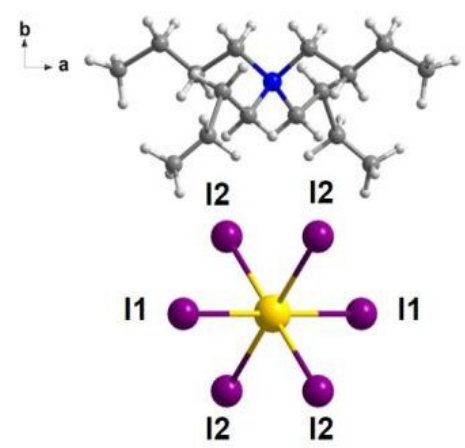

b
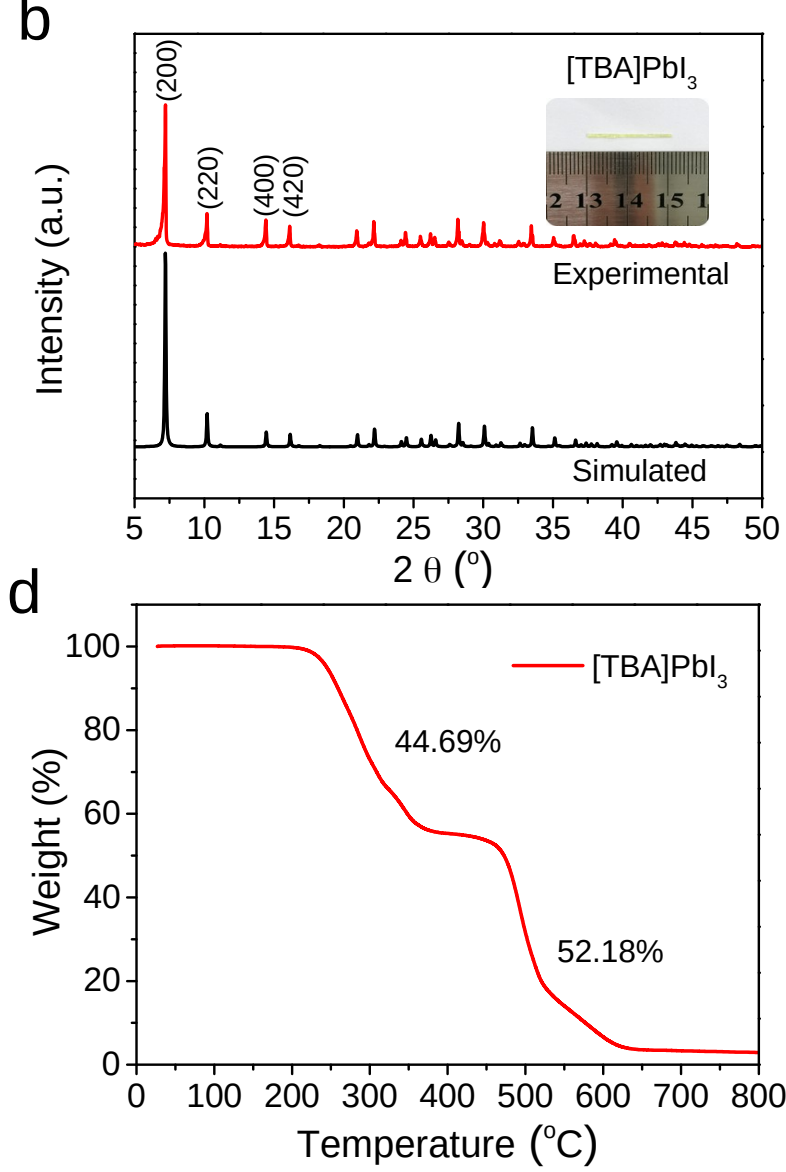

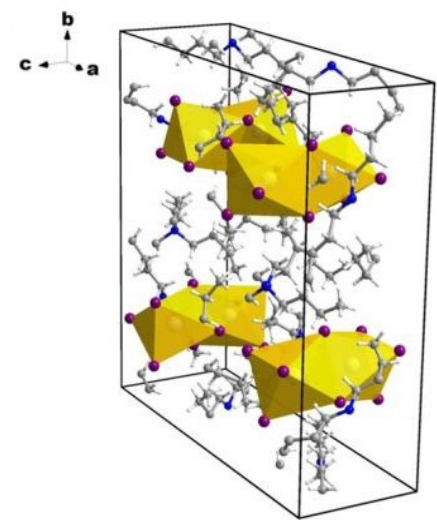

C

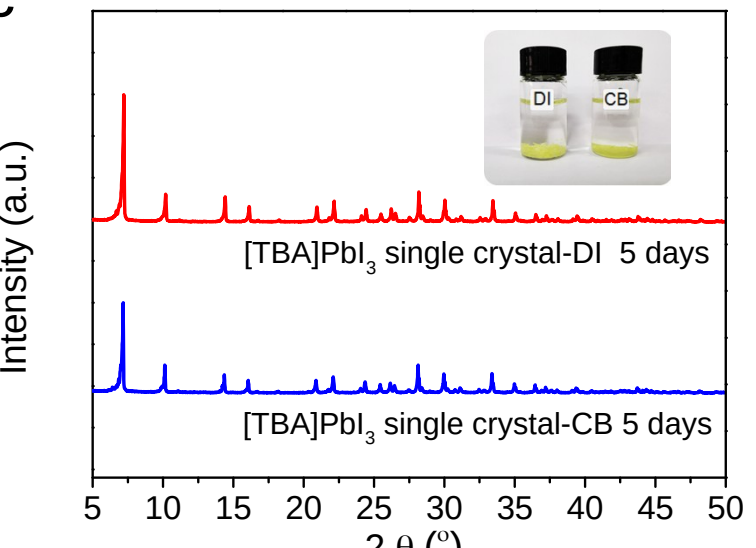

e

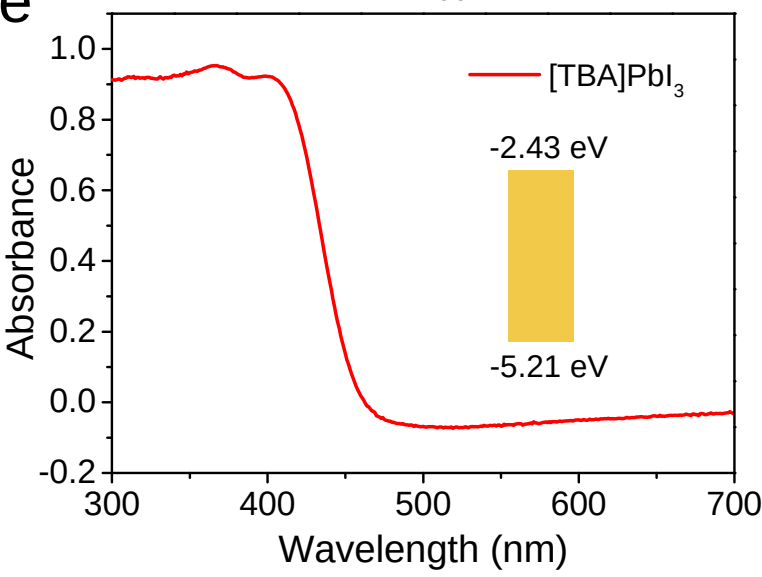

Figure 1. Characterization of $\left[\mathrm{TBA}^{\mathrm{P}} \mathrm{Pbl}_{3}\right.$ haloplumbate phase. a, Simulated and experimental X-ray diffraction (XRD) patterns of $[\mathrm{TBA}] \mathrm{Pbl}_{3}$ single crystals. The simulated pattern is based on the reported structure of [TBA] $\mathrm{Pbl}_{3}$ (CCDC no. 994586) ${ }^{38} \mathbf{~ b}$, Crystal structure of [TBA]Pbl ${ }_{3}$. $\mathbf{c}$, XRD patterns of [TBA]Pbl ${ }_{3}$ single crystals immersed into deionized water (DI) and chlorobenzene (CB) for 5 days. d, Thermogravimetric analysis of [TBA]Pbl ${ }_{3}$ single crystals. e, UV-vis absorption spectrum of [TBA]Pbl ${ }_{3}$. The inset shows the energy band gap of $[\mathrm{TBA}] \mathrm{Pbl}_{3}$ and the valence and conduction band positions. 
To assess the stability to moisture, the $[\mathrm{TBA}] \mathrm{Pbl}_{3}$ crystals were immersed into deionized (DI) water at ambient temperature. Moreover, since chlorobenzene $(C B)$ is used for depositing the charge transport layer, the stability in CB was also tested. The XRD patterns of [TBA]Pbl ${ }_{3}$ crystals in DI water and $\mathrm{CB}$ show no apparent change after 5 days (Figure 1c), suggesting that $[\mathrm{TBA}] \mathrm{Pbl}_{3}$ is stable under these conditions. The thermal stability was further tested by thermogravimetric analysis (TGA) to show that [TBA] $\mathrm{Pbl}_{3}$ remains stable below $200^{\circ} \mathrm{C}$ (Figure 1d), losing $44.69 \%$ weight only at $210-380^{\circ} \mathrm{C}$ and the remaining $52.18 \%$ weight at $380-640^{\circ} \mathrm{C}$, which corresponds to the volatilization of TBAI and the sublimation of lead iodide $\left(\mathrm{Pbl}_{2}\right)$, respectively. ${ }^{38}$ The TGA demonstrates excellent stability of $[\mathrm{TBA}] \mathrm{Pbl}_{3}$ against heat in the typical temperature range of perovskite device operation. Finally, the energy band structure of [TBA] $\mathrm{Pbl}_{3}$ was analyzed by the UV-vis diffuse reflection (Figure S2a and b) and the valence band X-ray photoelectron spectroscopy (VBXPS; Figure S3). The valence band maximum (VBM) of $[\mathrm{TBA}] \mathrm{Pbl}_{3}$ was found to be $5.21 \mathrm{eV}$ (Figure 1e; procedure described in the supporting information), between the VBM of widely used hybrid perovskite compositions and the highest occupied molecular orbital (HOMO) of 2,2',7,7'-tetrakis[ $N, N$-di(4methoxyphenyl)amino]-9,9'-spirobifluorene (Spiro-OMeTAD; Figure S4), which is beneficial for the hole extraction. ${ }^{47,48}$ In summary, $[\mathrm{TBA}] \mathrm{Pbl}_{3}$ has shown excellent water and thermal stability, as well as good energy band alignment, suggesting that it can be a suitable interfacial modulator.

Having demonstrated these excellent properties of [TBA]Pbl 3 , it was deposited onto the perovskite surface by using the solution of TBAI in chloroform $\left(\mathrm{CHCl}_{3}\right)$, a solvent that was shown not to affect the perovskite layer. ${ }^{49}$ This procedure results in the formation of the $[\mathrm{TBA}] \mathrm{Pbl}_{3}$ in-situ (experimental details are provided in the methods section). The mixed organic cation perovskite $\mathrm{FA}_{1-x} \mathrm{MA}_{\mathrm{x}} \mathrm{Pbl}_{3}$ was deposited by a two-step spin-coating method as control sample. ${ }^{22,50}$ The [TBA]Pbl ${ }_{3}$ layer is envisaged to exist on the surface of the perovskite layer without damaging the perovskite grains (Figure 2a). The top-view scanning electron microscopy (SEM) images (Figure $2 \mathrm{~b}$ and c) show an obvious change of perovskite film morphology after the treatment, which involves reducing the number of pinholes and grain boundaries that may cause shunts and stronger non-radiative recombination. Upon TBAI treatment, a thin layer with good coverage forms on the surface of the perovskite layer, which fills the pinholes and grain boundaries. This is in accordance with the atomic force microscopy (AFM) (Figure S5a, b) showing a reduced Root Mean Square (RMS) roughness from $33.5 \mathrm{~nm}$ to $21.3 \mathrm{~nm}$. To assess the structural properties of the layer, XRD measurements were performed. The XRD patterns (Figure 2d) show four new diffraction peaks at $7.2^{\circ}, 10.2^{\circ}, 14.44^{\circ}$, and $16.16^{\circ}$, corresponding to the $\langle 200\rangle,\langle 220\rangle,\langle 400\rangle$, and $\langle 420\rangle$ planes of $\left[\mathrm{TBA}_{\mathrm{Pbl}}\right.$, respectively. ${ }^{38}$ Furthermore, the intensity of the $\mathrm{Pbl}_{2}$ peak at $12.6^{\circ}$ decreases after TBAI treatment upon formation of [TBA] $\mathrm{Pbl}_{3}$ comes through the reaction with $\mathrm{Pbl}_{2}$. This is further evidenced by the XRD patterns of the $\mathrm{Pbl}_{2}$ films treated with TBAI (Figure S6), which revealed the formation of [TBA]Pbl ${ }_{3}$ under these conditions. 2D grazing-incidence X-ray diffraction (GIXRD) patterns (Figure $2 e, f)$ show four new scattering rings upon TBAI treatment, evidencing formation of $1 \mathrm{D}[\mathrm{TBA}] \mathrm{Pbl}_{3}$ on the surface of perovskite layer in random directions parallel to the substrate. 
a

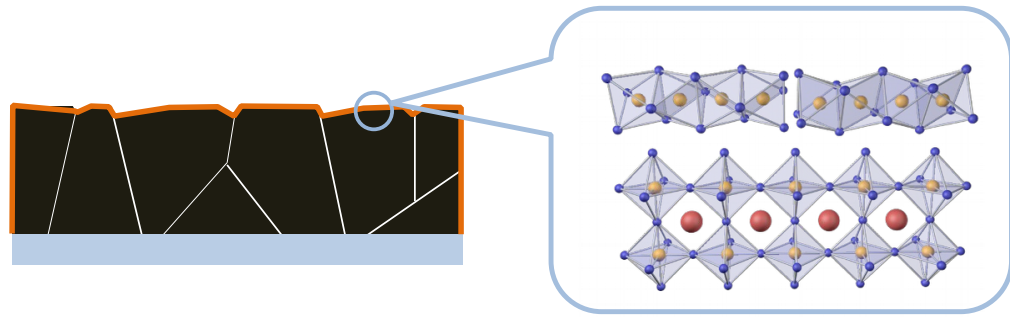

b
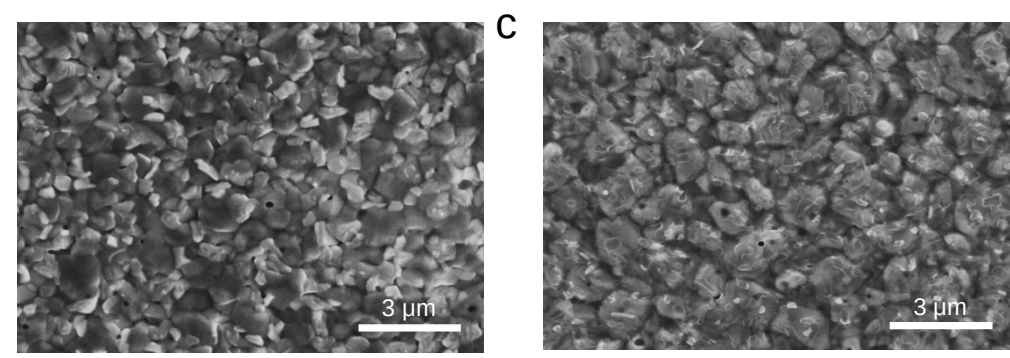

$\mathrm{e}$
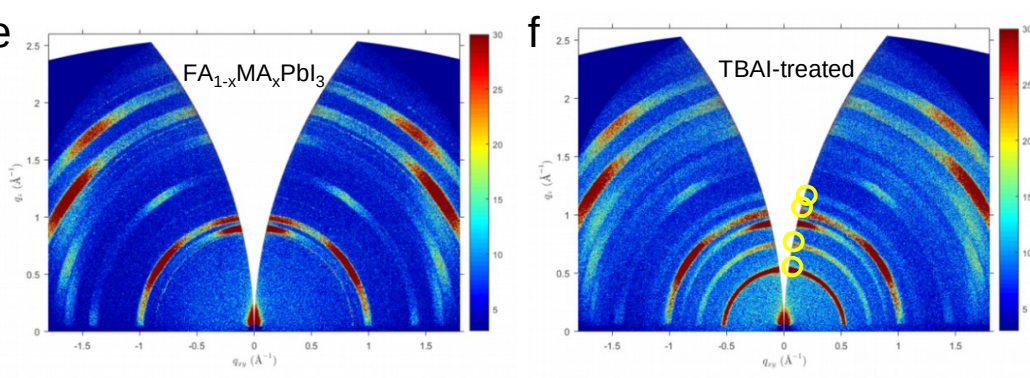
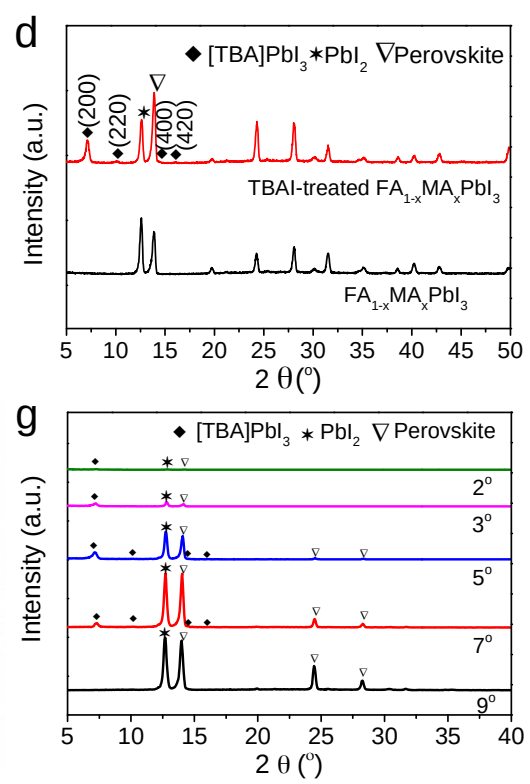

Figure 2. Modulated perovskite film characterization. a, Simplified scheme presenting the perovskite films modulated with [TBA]Pbl 3 . b-c, Top-view scanning electron microscopy (SEM) images of the $\mathrm{FA}_{1-\times} \mathrm{MA}_{\times} \mathrm{Pbl}_{3}$ and TBAI-treated perovskite films. $\mathbf{d}, \mathrm{XRD}$ patterns of the $\mathrm{FA}_{1-\mathrm{X}} \mathrm{MA}_{\mathrm{x}} \mathrm{Pbl}_{3}$ and TBAI-treated perovskite films. e-f, Two-dimensional (2D) grazing-incidence X-ray diffraction (GIXRD) patterns of $\mathrm{FA}_{1-\times} \mathrm{MA}_{\times} \mathrm{Pbl}_{3}$ and TBAI-treated perovskite films (yellow circles in $\mathrm{f}$ indicate the formation of new signals). $\mathbf{g}$, GIXRD patterns of TBAl-treated perovskite films at different incidence angles.

Interestingly, the reaction between the perovskite and TBAI was found to be self-limiting. In general, when depositing a 2D perovskite overlayer through an in-situ reaction process, the larger organic cations exchange with the smaller cations in the perovskite layer continuously until the entire $3 \mathrm{D}$ perovskite layer is converted to $2 \mathrm{D}$ perovskite. ${ }^{51}$ In contrast, the reaction between perovskite and TBAl stops after forming a thin layer of $\left[\mathrm{TBA}_{\mathrm{Pb}} \mathrm{B}_{3}\right.$. To demonstrate this, we immersed three perovskite films into isopropanol (IPA) and the solutions of either $n$-butylammonium iodide (BAI) or TBAI in IPA (Figure S7). The BAI was employed as a widely used organic cation for 2D perovskites. The edge of the film in IPA turns yellow after 6 hours as the perovskite layer suffers from degradation in IPA, whereas the film immersed in the solution of BAI completely turns yellow after the same period of time. The XRD patterns of the corresponding films (Figure S8a) show intense $\mathrm{Pbl}_{2}$ peaks and weaker perovskite peaks over time, indicating that the cation exchange process accelerates the degradation of the film. However, the film placed in the TBAI solution remains black even after $24 \mathrm{~h}$, and the corresponding XRD patterns maintain the original intensity (Figure S8b), indicating that the reaction is self-limiting. 
As a result, the TBAI treatment is supposed to influence the surface of the perovskite without affecting the bulk. To probe this effect on the distribution of [TBA]Pb| ${ }_{3}$, we analyzed GIXRD with different incidence angles (Figure $2 \mathrm{~g}$ ). With the increase of the incidence angles, the perovskite peak intensities increase, yet the $[\mathrm{TBA}] \mathrm{Pbl}_{3}$ peaks become stronger for incidence angles up to $5^{\circ}$, after which they become weaker and disappear with further increase of the angle of incidence. This suggests that $[\mathrm{TBA}] \mathrm{Pbl}_{3}$ mainly exists on the surface of the perovskite layer ${ }^{52,53}$, which is further analyzed by X-ray photoelectron spectroscopy (XPS). The XPS spectra of perovskite films show shifts of peak positions in the $\mathrm{N}$ 1s core level range (Figure S9a), featuring two peaks at binding energies of $400.15 \mathrm{eV}$ and $400.45 \mathrm{eV}$, corresponding to the $\mathrm{N}$ atoms in the formamidinium (FA $\left.{ }^{+}\right) \mathrm{A}$ cation $^{54}$. In the [TBA] $\mathrm{Pbl}_{3}$-modulated sample, there is only one peak at $401.38 \mathrm{eV}$ corresponding to $\mathrm{N}\left(\mathrm{C}_{4} \mathrm{H}_{9}\right)_{4}{ }^{+}$, which indicates complete transformation of the perovskite to [TBA]Pbl${ }_{3}$ on the surface. The $\mathrm{Pb} 4 \mathrm{f}$ and $\mathrm{C}$ 1s core level spectra (Figure S9b, c) also show apparent peak shifts upon [TBA] $\mathrm{Pbl}_{3}$ modulation. In addition, the $\mathrm{C}=\mathrm{O}$ signal at $288.05 \mathrm{eV}$ in the $\mathrm{C} 1 \mathrm{~s}$ spectra (Figure $\mathrm{S} 9 \mathrm{c}$ ) of the control sample disappears upon TBAl treatment, which suggests that TBAPbl ${ }_{3}$ could protect perovskite from moisture and oxygen in the air $^{22}$. To prove this effect, we immersed the perovskite films into DI water (Movie S1 and S2, supporting information). While the control film completely turns yellow after 7 seconds, the [TBA]Pbl ${ }_{3}$-modulated film (obtained by immersing the perovskite film into TBAI solution) remains black in 1 minute, which indicates that the [TBA]Pbl ${ }_{3}$ interlayer could have a protective effect.

We thereafter investigated optical and electronic properties of the [TBA]Pbl${ }_{3}$-modulated films and devices. The steady state photoluminescence $(P L)$ spectroscopy (Figure 3a) shows a fivefold enhancement in $\mathrm{PL}$ intensity upon TBAl treatment, suggesting that $[\mathrm{TBA}] \mathrm{Pbl}_{3}$ might passivate the defects of perovskite film to suppress the non-radiative recombination. Accordingly, time-resolved photoluminescence (TRPL) spectroscopy (Figure $3 \mathrm{~b}$ ) reveals an increase in charge carrier lifetimes from 500.01 ns to 1049.03 ns (Table S1, supporting information), whereas confocal PL mapping of control and $[\mathrm{TBA}] \mathrm{Pbl}_{3}$-modulated perovskite films (Figure $3 \mathrm{c}$ ) shows an increase in the $\mathrm{PL}$ brightness of the surface from 120.60 to 155.26 after TBAI treatment. This is likely caused by the passivation of defects on the surface and at the grain boundaries of the perovskite. ${ }^{55}$ To quantify the electron-trap densities $\left(n_{t}\right)$ in the control and [TBA]Pbl ${ }_{3}$-modulated devices, the space-chargelimited-current (SCLC) measurements were performed (experimental details are provided in the Experimental Section) with an electron-only device structure of $I T O / \mathrm{SnO}_{2} / \mathrm{FA}_{1-x} \mathrm{MA}_{x} \mathrm{Pbl}_{3} / \mathrm{PCBM} / \mathrm{Au}$ (ITO for indium tin oxide; Figure S10). ${ }^{50,56}$ The trap filled limit voltage ( $\left.V_{\mathrm{TFL}}\right)$ decreases from $1.050 \mathrm{~V}$ to $0.625 \mathrm{~V}$, corresponding to the reduced density of trap states in the perovskite film from $9.9 \times 10^{15}$ $\mathrm{cm}^{-3}$ to $5.9 \times 10^{15} \mathrm{~cm}^{-3}$ upon TBAl treatment, which is particularly relevant for the photovoltaic performances. $^{47,50}$ 

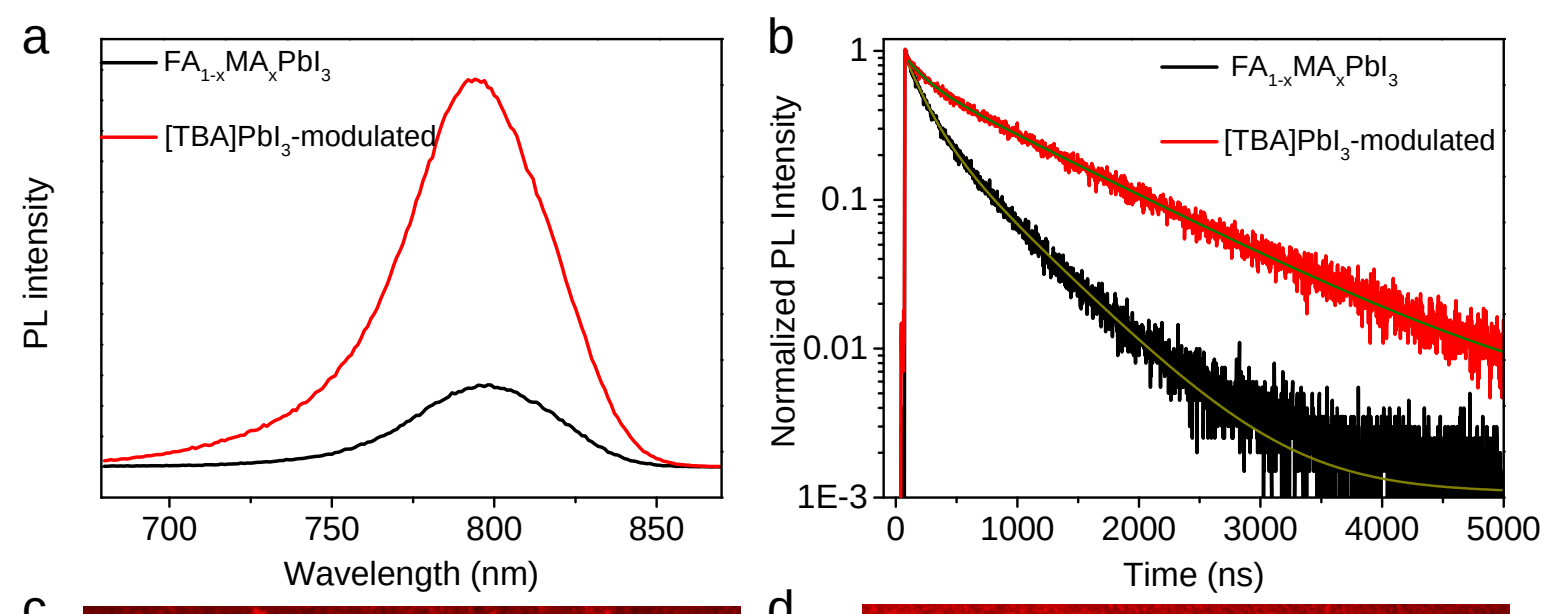

C
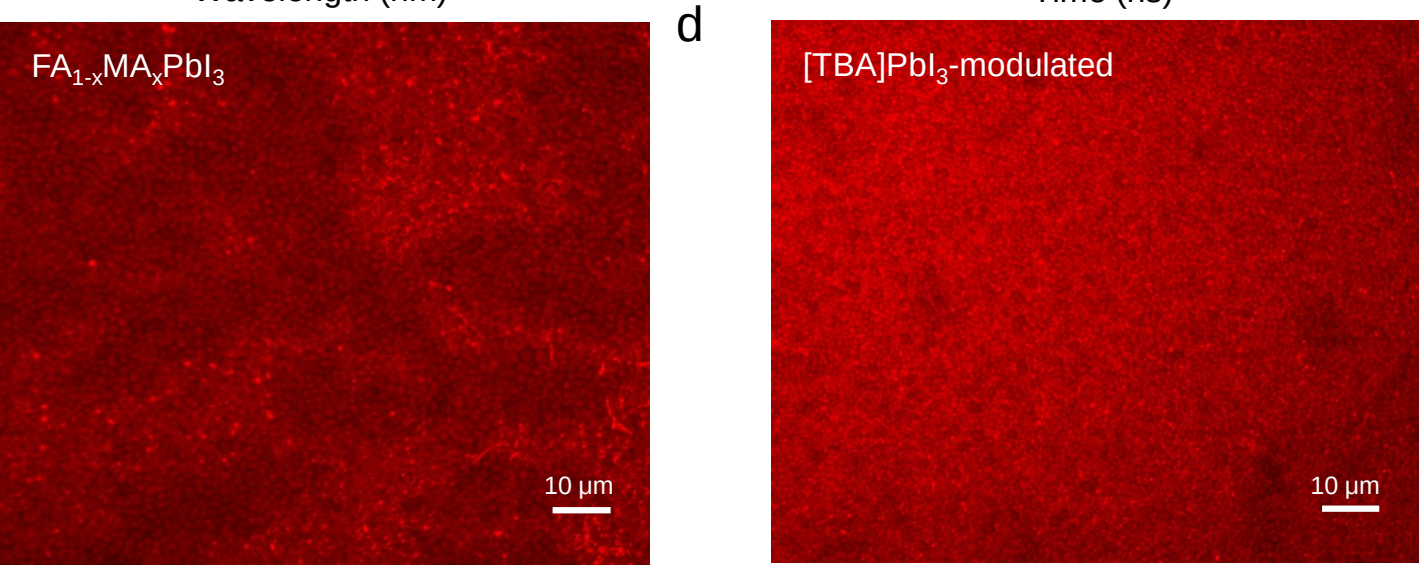

Figure 3. Photoluminescence of modulated perovskite films. a, Steady state $\mathrm{PL}$ spectra of $F A_{1-x} M A_{x} P b l_{3}$ and [TBA]Pbl3-modulated perovskite films. $\mathbf{b}$, Time-resolved photoluminescence spectra of $\mathrm{FA}_{1-x} \mathrm{MA}_{x} \mathrm{Pbl}_{3}$ and [TBA]Pbl ${ }_{3}$-modulated perovskite films. $\mathbf{c}$, $\mathbf{d}$, Confocal $\mathrm{PL}$ mapping of the intensity on $\mathrm{FA}_{1-x} \mathrm{MA}_{x} \mathrm{Pbl}_{3}$ and [TBA]PbI3-modulated perovskite films.

The photovoltaic devices were fabricated with the ITO/SnO $/ / \mathrm{FA}_{1-x} \mathrm{MA}_{x} \mathrm{Pbl} / \mathrm{I}_{3} /$ spiro-OMeTAD/Au architecture as our previous report. ${ }^{50}$ The best device demonstrated the efficiency of $22.90 \%$ $\left(V_{\mathrm{OC}}=1.15 \mathrm{~V}, J_{\mathrm{SC}}=24.81 \mathrm{~mA} \mathrm{~cm}{ }^{-2}, \mathrm{FF}=80.27 \%\right)$, which has been improved as compared to the control device with an efficiency of $21.53 \%\left(V_{\mathrm{OC}}=1.12 \mathrm{~V}, \mathrm{~J}_{\mathrm{SC}}=24.80 \mathrm{~mA} \mathrm{~cm}^{-2}, \mathrm{FF}=77.64 \%\right)$. In addition, the $[\mathrm{TBA}] \mathrm{Pbl}_{3}$-modulated device showed a lower hysteresis factor compared to control device (Figure S11 and Table S2). The average efficiency and repeatability were also improved (Figure S12), whereas the integrated current density calculated from the external quantum efficiency (EQE) was found to be $23.94 \mathrm{~mA} \mathrm{~cm}^{-2}$ (Figure 4b), which is consistent with the current-voltage (J-V) measurements. This improvement in the photovoltaic performances is likely related to the passivation effect of $[\mathrm{TBA}] \mathrm{Pbl}_{3}$.

To further study the dynamics of carriers, we analyzed the transient photovoltage decay (TPV). TPV decay curves (Figure $4 \mathrm{c}$ ) show a higher recombination constant $\left(\tau_{\mathrm{r}}\right)$, from $2.87 \mu \mathrm{s}$ to $6.45 \mu \mathrm{s}$, in accordance with the slower surface recombination in the modulated devices. Electrical impedance spectroscopy (EIS) spectra (Figure S13) also corroborated with the reduced non-radiative 
recombination, showing increased recombination resistance $\left(\mathrm{R}_{\mathrm{re}}\right)$ of the $[\mathrm{TBA}] \mathrm{Pbl}_{3}$-modulated device from $2736 \Omega$ to $3646 \Omega$ (Table S3)..$^{57,58}$
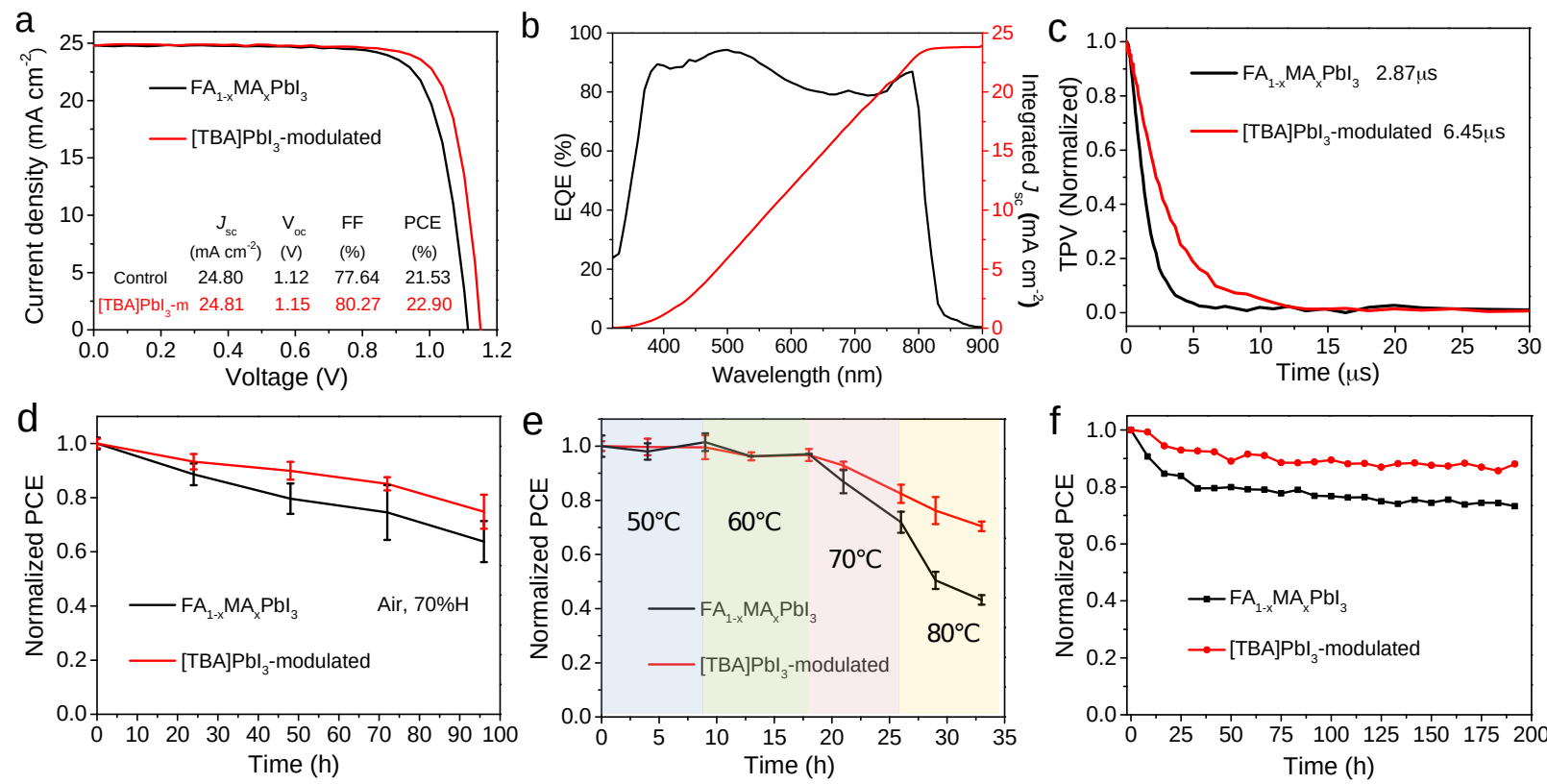

Figure 4. Device performance and stability. a, Current density-voltage ( $\mathrm{J}-\mathrm{V})$ curves of $\mathrm{FA}_{1-x} \mathrm{MA}_{\times} \mathrm{Pbl}_{3}$ and $[\mathrm{TBA}] \mathrm{Pbl}_{3}$-modulated devices. b, External quantum efficiency (EQE) spectra of [TBA]Pbl${ }_{3}$-modulated device. c, Normalized transient photovoltage (TPV) decay curves of $\mathrm{FA}_{1-x} \mathrm{MA}_{\mathrm{x}} \mathrm{Pbl}_{3}$ and [TBA]Pbl${ }_{3}$-modulated devices. d, Normalized power conversion efficiency (PCE) of devices stored in the air with $70 \%$ relatively humidity (RH) under dark and $\mathbf{e}$, Normalized PCE of devices stored in nitrogen-filled glove box under elevated temperatures, five control devices and five [TBA]Pbl${ }_{3}$-modulated devices were tested, the error bars were based on results of five devices, and they are the standard deviations. f, Normalized PCE of devices measured by maximum power point tracking (MPPT) under 1 sun illumination at room temperature.

Considering the self-limiting feature of the $[\mathrm{TBA}] \mathrm{Pbl}_{3}$ layer, devices with the perovskite layer immersed in the TBAl solution in $\mathrm{CHCl}_{3}$ for different periods of time show almost identical $\mathrm{J}-\mathrm{V}$ curves, evidencing that the immersion time does not influence the device performance (Figure S14 and Table S4). To exclude the effect of the solvent used, we compared the performances of the control devices, the $\mathrm{CHCl}_{3}$ modulated devices, and the TBAl/ $\mathrm{CHCl}_{3}$ solution treated ones (Figure S15). The results show that the $\mathrm{CHCl}_{3}$ modulated devices have no increase in efficiency and repeatability.

We thereby investigated the humidity, thermal, operational, and long-term stability of [TBA]Pbl ${ }_{3}$ modulated devices. We exposed the control and [TBA]Pbl ${ }_{3}$-modulated perovskite films to air at $75 \%$ relative humidity $(\mathrm{RH})$ at ambient temperature and monitored their changes. While the control film decomposed after $48 \mathrm{~h}$, the [TBA]Pb| ${ }_{3}$-modulated film featured no visual change after $120 \mathrm{~h}$ (Figure S16). Accordingly, the efficiencies of [TBA]Pb/ ${ }_{3}$-modulated devices show higher resilience than the control devices in the moist air (Figure 4d). The thermal stability of the [TBA]Pbl${ }_{3}$-modulated devices is also improved, especially at temperatures above $70{ }^{\circ} \mathrm{C}$ (Figure 4e). The [TBA]Pbl ${ }_{3}$-modulated perovskite films show a lower decomposition rate (Figure S17) under environmental conditions and 
thermal stress. In addition, the efficiencies of devices show no change under long-term storage (Figure S18). Under continuous maximum power point tracking operation, the [TBA]Pbl ${ }_{3}$-modulated device maintains $86 \%$ efficiency after 200 hours while the efficiency of the control device reduced significantly (Figure 4f).

\section{Conclusion}

In summary, we have introduced haloplumbate materials based on tetra-butylammonium (TBA) organic moieties as the interfacial modulator for PSCs. The haloplumbate [TBA]Pbl ${ }_{3}$ forms in-situ at the interface between perovskite and hole transport layer with excellent stability and matched band energy level alignment. The modulated perovskite samples show reduced trap density and increased stability. As a result, the champion photovoltaic device has a power conversion efficiency of $22.90 \%$, along with enhanced stability under environmental and operational conditions, as well as long-term storage. The remarkable stability and the favorable effects on perovskite materials as well as the ease of fabrication render haloplumbate-based interfacial modulators ideal candidates for addressing the stability problem that impedes the commercialization of PSCs.

\section{Supporting Information}

Materials, experimental methods, Figure S1-S18, Table S1-S4, and Movie S1-S2.

\section{Acknowledgements}

The authors would like to thank Dr. Ke Meng for the GIXRD characterization. This study was supported by the National Key Research and Development Program of China (Grant No. 2018YFB1502003; 2019YFE0123400), the Tianjin Distinguished Young Scholars Fund (20JCJQJC00260), the Chinese Thousand Talents Program for Young Professionals, the "111" Project (Grant No. B16027), National Natural Science Foundation of China (Grant No. 21872080), and the startup funding of Nankai University. J.V.M. is grateful for the Swiss National Science Foundation no. 193174.

\section{Author contributions}

J.L. conceived and designed the project. H.W. and Z.Z. fabricated and characterized devices. J.M. and Z.W. provided guidance for the project. L.T. performed EQE, TPV, and stability measurement, along with analyzing the data. R.C. analyzed the structure of single crystal. X.J. conducted PL, TRPL and UV-vis test. The manuscript was written by H.W., Z.Z., and J. L. with contributions from all authors. J.L. supervised the project. All authors discussed the results and contributed to the work.

\section{Reference}


1 Rong, Y. et al. Challenges for commercializing perovskite solar cells. Science 361 (2018).

2 Jena, A. K., Kulkarni, A. \& Miyasaka, T. Halide Perovskite Photovoltaics: Background, Status, and Future Prospects. Chem Rev 119, 3036-3103 (2019).

3 Turren-Cruz, S. H., Hagfeldt, A. \& Saliba, M. Methylammonium-free, high-performance, and stable perovskite solar cells on a planar architecture. Science 362, 449-453 (2018).

4 Jung, E. H. et al. Efficient, stable and scalable perovskite solar cells using poly(3hexylthiophene). Nature 567, 511-515 (2019).

$5 \mathrm{Nie}, \mathrm{W}$. et al. Solar cells. High-efficiency solution-processed perovskite solar cells with millimeter-scale grains. Science 347, 522-525 (2015).

$6 \mathrm{Kim}, \mathrm{G}$. et al. Impact of strain relaxation on performance of alpha-formamidinium lead iodide perovskite solar cells. Science 370, 108-112 (2020).

7 Lu, H. et al. Vapor-assisted deposition of highly efficient, stable black-phase $\mathrm{FAPbl}_{3}$ perovskite solar cells. Science $\mathbf{3 7 0}$ (2020).

8 Kojima, A., Teshima, K., Shirai, Y. \& Miyasaka, T. Organometal halide perovskites as visiblelight sensitizers for photovoltaic cells. J Am Chem Soc 131, 6050-6051 (2009).

9 Best Research-Cell Efficiencies (NREL, 2021); https://www.nrel.gov/pv/cell-efficiency.html.

10 Stranks, S. D. et al. Electron-hole diffusion lengths exceeding 1 micrometer in an organometal trihalide perovskite absorber. Science 342, 341-344 (2013).

11 Dong, Q. et al. Solar cells. Electron-hole diffusion lengths > 175 mum in solution-grown $\mathrm{CH}_{3} \mathrm{NH}_{3} \mathrm{Pbl}_{3}$ single crystals. Science 347, 967-970 (2015).

12 Lin, K. et al. Perovskite light-emitting diodes with external quantum efficiency exceeding 20 per cent. Nature 562, 245-248 (2018).

13 Gratzel, M. The light and shade of perovskite solar cells. Nat Mater 13, 838-842 (2014).

14 Yang, S. et al. Functionalization of perovskite thin films with moisture-tolerant molecules. Nature Energy 1 (2016).

15 Leijtens, T. et al. Stability of Metal Halide Perovskite Solar Cells. Advanced Energy Materials 5 (2015).

16 Wang, R. et al. A Review of Perovskites Solar Cell Stability. Advanced Functional Materials 29 (2019).

17 Hoke, E. T. et al. Reversible photo-induced trap formation in mixed-halide hybrid perovskites for photovoltaics. Chem Sci 6, 613-617 (2015).

18 Wei, J. et al. Mechanisms and Suppression of Photoinduced Degradation in Perovskite Solar Cells. Advanced Energy Materials 11 (2020).

19 Shao, S. \& Loi, M. A. The Role of the Interfaces in Perovskite Solar Cells. Advanced Materials Interfaces 7 (2019).

20 Milic, J. V., Kubicki, D. J., Emsley, L. \& Gratzel, M. Multifunctional Molecular Modulation for Efficient and Stable Hybrid Perovskite Solar Cells. Chimia (Aarau) 73, 317-323 (2019).

21 Zhang, H., Nazeeruddin, M. K. \& Choy, W. C. H. Perovskite Photovoltaics: The Significant Role of Ligands in Film Formation, Passivation, and Stability. Adv Mater 31, e1805702 (2019).

22 Jiang, Q. et al. Surface passivation of perovskite film for efficient solar cells. Nature Photonics 13, 460-466 (2019).

23 Wang, R. et al. Constructive molecular configurations for surface-defect passivation of $10 / 12$ 
perovskite photovoltaics. Science 366, 1509-1513 (2019).

24 Milić, J. V., Arora, N., Dar, M. I., Zakeeruddin, S. M. \& Grätzel, M. Reduced Graphene Oxide as a Stabilizing Agent in Perovskite Solar Cells. Advanced Materials Interfaces 5 (2018).

25 Alharbi, E. A. et al. Atomic-level passivation mechanism of ammonium salts enabling highly efficient perovskite solar cells. Nat Commun 10, 3008 (2019).

26 Liu, Y. et al. Ultrahydrophobic 3D/2D fluoroarene bilayer-based water-resistant perovskite solar cells with efficiencies exceeding 22. Sci Adv 5, eaaw2543 (2019).

$27 \mathrm{Bi}, \mathrm{D}$. et al. Polymer-templated nucleation and crystal growth of perovskite films for solar cells with efficiency greater than 21\%. Nature Energy 1 (2016).

28 Yang, S. et al. Stabilizing halide perovskite surfaces for solar cell operation with wide-bandgap lead oxysalts. Science 365, 473-478 (2019).

29 Xue, C. et al. Extra thermo- and water-stable one-dimensional organic-inorganic hybrid perovskite [N-methyldabconium] $\mathrm{Pbl}_{3}$ showing switchable dielectric behaviour, conductivity and bright yellow-green emission. Chem Commun (Camb) 54, 4321-4324 (2018).

30 Liao, W. Q. et al. A lead-halide perovskite molecular ferroelectric semiconductor. Nat Commun 6, 7338 (2015).

31 Garcia-Fernandez, A. et al. $\left[\left(\mathrm{CH}_{3}\right)_{2} \mathrm{NH}_{2}\right]_{7} \mathrm{~Pb}_{4} \mathrm{X}_{15}\left(\mathrm{X}=\mathrm{Cl}\left({ }^{-}\right)\right.$and $\left.\mathrm{Br}\left({ }^{-}\right)\right)$, 2D-Perovskite Related Hybrids with Dielectric Transitions and Broadband Photoluminiscent Emission. Inorg Chem 57, 3215-3222 (2018).

32 Garcia-Fernandez, A. et al. Phase Transition, Dielectric Properties, and Ionic Transport in the $\left[\left(\mathrm{CH}_{3}\right)_{2} \mathrm{NH}_{2}\right] \mathrm{Pbl}_{3}$ Organic-Inorganic Hybrid with $2 \mathrm{H}$-Hexagonal Perovskite Structure. Inorg Chem 56, 4918-4927 (2017).

33 Zhang, W. F. et al. One-Dimensional Face-Shared Perovskites with Broad-Band Bluish WhiteLight Emissions. Inorg Chem 59, 14085-14092 (2020).

34 Yuan, Z. et al. One-dimensional organic lead halide perovskites with efficient bluish white-light emission. Nat Commun 8, 14051 (2017).

$35 \mathrm{Wu}, \mathrm{Z}$. et al. Broad-Band-Emissive Organic-Inorganic Hybrid Semiconducting Nanowires Based on an $\mathrm{ABX}_{3}$-Type Chain Compound. Inorg Chem 56, 8776-8781 (2017).

36 Shi, M., Yu, S.-S., Zhang, H., Liu, S.-X. \& Duan, H.-B. A hybrid molecular rotor crystal with dielectric relaxation and thermochromic luminescence. Journal of Molecular Structure 1206 (2020).

37 Duan, H.-B., Yu, S.-S., Liu, S.-X. \& Zhang, H. A multi-functional iodoplumbate-based hybrid crystal: 1-propyl-4-aminopyridinium triiodoplumbate. RSC Advances 7, 23234-23237 (2017).

38 She, Y.-J., Zhao, S.-P., Tian, Z.-F. \& Ren, X.-M. Inorganic-organic hybrid with one-dimensional face-sharing iodoplumbate chain showing novel dielectric anomaly and semiconductor emission. Inorganic Chemistry Communications 46, 29-32 (2014).

39 Song, X. et al. Overall photocatalytic water splitting by an organolead iodide crystalline material. Nature Catalysis 3, 1027-1033 (2020).

$40 \mathrm{Li}, \mathrm{H}$. et al. Perovskite films with a sacrificial cation for solar cells with enhanced stability based on carbon electrodes. Journal of Alloys and Compounds 797, 811-819 (2019).

41 Jin, S. et al. Improving perovskite solar cells photovoltaic performance using tetrabutylammonium salt as additive. Journal of Power Sources 450 (2020). 
42 Poli, I., Eslava, S. \& Cameron, P. Tetrabutylammonium cations for moisture-resistant and semitransparent perovskite solar cells. J. Mater. Chem. A 5, 22325-22333 (2017).

$43 \mathrm{Xu}, \mathrm{Z}$. et al. Br-containing alkyl ammonium salt-enabled scalable fabrication of high-quality perovskite films for efficient and stable perovskite modules. Journal of Materials Chemistry A 7 , 26849-26857 (2019).

44 Kour, P., Chenna Reddy, M., Naphade, R. \& Ogale, S. Quaternary alkylammonium salt incorporated 2D/3D mixed halide perovskite with highly enhanced photoluminescence and arrested iodide/bromide phase segregation. APL Materials 6 (2018).

$45 \mathrm{Liu}, \mathrm{X}$. et al. Organic Tetrabutylammonium Cation Intercalation to Heal Inorganic $\mathrm{CsPbl}_{3}$ Perovskite. Angew Chem Int Ed Engl (2021).

46 Kang, D.-H., Kim, S.-Y., Lee, J.-W. \& Park, N.-G. Efficient surface passivation of perovskite films by a post-treatment method with a minimal dose. Journal of Materials Chemistry $A \mathbf{9}$, 3441-3450 (2021).

47 Zhou, T. et al. Highly Efficient and Stable Solar Cells Based on Crystalline Oriented 2D/3D Hybrid Perovskite. Adv Mater 31, e1901242 (2019).

48 Jiang, Q. et al. Enhanced electron extraction using $\mathrm{SnO}_{2}$ for high-efficiency planar-structure $\mathrm{HC}\left(\mathrm{NH}_{2}\right)_{2} \mathrm{Pbl}_{3}$-based perovskite solar cells. Nature Energy 2 (2016).

49 Yoo, J. J. et al. An interface stabilized perovskite solar cell with high stabilized efficiency and low voltage loss. Energy \& Environmental Science 12, 2192-2199 (2019).

50 Wang, $\mathrm{H}$. et al. Ligand-Modulated Excess $\mathrm{Pbl}_{2}$ Nanosheets for Highly Efficient and Stable Perovskite Solar Cells. Adv Mater 32, e2000865 (2020).

51 Lin, Y. et al. Enhanced Thermal Stability in Perovskite Solar Cells by Assembling 2D/3D Stacking Structures. J Phys Chem Lett 9, 654-658 (2018).

52 Zhang, Y. et al. $\mathrm{Pbl}_{2}$-HMPA Complex Pretreatment for Highly Reproducible and Efficient CH3NH3Pbl3 Perovskite Solar Cells. J Am Chem Soc 138, 14380-14387 (2016).

53 Liu, J. et al. Self-formed $\mathrm{Pbl}_{2}$-DMSO adduct for highly efficient and stable perovskite solar cells. Applied Physics Letters 115 (2019).

54 Zhuang, J. et al. Interfacial Passivation for Perovskite Solar Cells: The Effects of the Functional Group in Phenethylammonium lodide. ACS Energy Letters 4, 2913-2921 (2019).

55 Xin, C. et al. Defects Healing in Two-Step Deposited Perovskite Solar Cells via Formamidinium Iodide Compensation. ACS Applied Energy Materials 3, 3318-3327 (2020).

56 Yang, X. et al. Superior Carrier Lifetimes Exceeding 6 micros in Polycrystalline Halide Perovskites. Adv Mater 32, e2002585 (2020).

$57 \mathrm{Hou}, \mathrm{M}$. et al. Aryl Diammonium Iodide Passivation for Efficient and Stable Hybrid OrganInorganic Perovskite Solar Cells. Advanced Functional Materials 30 (2020).

58 Wang, P. et al. Gradient Energy Alignment Engineering for Planar Perovskite Solar Cells with Efficiency Over 23. Adv Mater 32, e1905766 (2020). 